Review

\title{
The emerging function and clinical significance of circRNAs in Thyroid Cancer and Autoimmune Thyroid Diseases
}

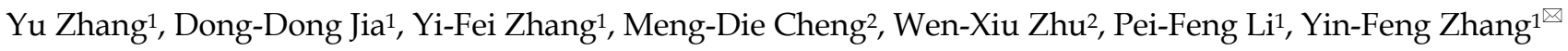 \\ 1. Institute for Translational Medicine, The Affiliated Hospital of Qingdao University, College of Medicine, Qingdao University, Deng Zhou Road 38, Qingdao 266021, \\ China. \\ 2. Department of Cardiology, The Affiliated Hospital of Qingdao University, Qingdao, China. Institute for Translational Medicine, Qingdao University, Qingdao, China. \\ $凶$ Corresponding author: Yin-Feng Zhang, E-mail: zhangyinfeng@qdu.edu.cn.
}

(c) The author(s). This is an open access article distributed under the terms of the Creative Commons Attribution License (https://creativecommons.org/licenses/by/4.0/). See http://ivyspring.com/terms for full terms and conditions.

Received: 2020.11.02; Accepted: 2021.04.07; Published: 2021.04.17

\begin{abstract}
Thyroid cancer (TC) is one of the most common malignant tumors, with high morbidity and mortality rates worldwide. The incidence of TC, especially that of papillary thyroid carcinoma (PTC); has increased rapidly in recent decades. Autoimmune thyroid disease (AITD) is closely related to TC and has an estimated prevalence of $5 \%$. Thus, it is becoming increasingly important to identify potential diagnostic biomarkers and therapeutic targets for TC and AITD. Circular RNAs (circRNAs) are a class of non-coding RNAs with covalently bonded circular structures that lack 5'-3' polarity and polyadenylated tails. Several circRNAs play crucial roles in the development of various diseases, including TC and AITD, and could be important new biomarkers and/or targets for the diagnosis and therapy of such disorders. Although there are four subtypes of TC, research on circRNA has largely focused on its connection to PTC. Therefore, this review mainly summarizes the relationships between circRNAs and PTC and AITD, including the molecular mechanisms underlying these relationships. In particular, the functions of "miRNA sponges" and their interactions with proteins and RNA are discussed. The possible targeting of circRNAs for the prevention, diagnosis, and treatment of TC and AITD is also described. CircRNAs could be potential biomarkers of TC and AITD, although validation will be required before they can be implemented in clinical practice.
\end{abstract}

Key words: circular RNA; thyroid cancer; autoimmune thyroid diseases; biomarker; therapeutic target

\section{Introduction}

\subsection{Thyroid cancer and autoimmune thyroid diseases}

Thyroid cancer (TC) has long been considered to arise in middle age and, after repeated proliferation, resulting in further damage to the genome, can progress to more aggressive and lethal types of cancer [1]. Three major studies published in 2014 led to a significant change in our understanding of the natural history of TC [1-5]. These studies, known as the 2014 TC trilogy, were the first to report the existence of self-limiting cancers [6-10]. There are four subtypes of TC, namely papillary thyroid carcinoma (PTC), follicular thyroid carcinoma, medullary thyroid carcinoma, and anaplastic thyroid carcinoma. PTC accounts for about $80 \%$ of TC followed by follicular carcinoma, which accounts for about 10\% [11]. Autoimmune thyroid diseases (AITDs), which are related to TC [12], are caused by a dysregulation of the immune system that results in an immune attack on the thyroid. AITDs such as chronic lymphocytic thyroiditis and Graves' disease (GD) are B cell- and T cell-mediated organ-specific autoimmune disorders $[13,14]$. Chronic lymphocytic thyroiditis was first proposed by the Japanese scholar Hashimoto in 1912, hence it is also known as Hashimoto's thyroiditis (HT) [15].

Although TC was once considered a relatively uncommon malignancy, in general, the incidence of TC has been increasing year by year due to various 
potential factors including environmental contaminations [16, 17]. Indeed, long-term, highquality registry data have revealed that the incidence of the disease has increased substantially over the past few decades in almost every country [11, 18]. Approximately 567,000 cases of TC are reported worldwide, and the disease was ranked in ninth place for worldwide cancer incidence in 2018 [19]. Throughout the world, the incidence of TC is approximately three times higher in women (6.1 per 100,000 per year) than in men (1.9 per 100,000 per year) [20]. Initially, palpation was the predominant means of finding thyroid nodules, and surgery was necessary to diagnose TC [21]. Later, technical improvements (including the development of new forms of tracers and pin-hole collimation) enabled thyroid scans to become more sensitive than palpation [22]. The most recent advance in thyroid imaging is the use of high-definition ultrasonography; however, although this technique is highly sensitive, it lacks specificity because most thyroid nodules are benign, and very small cancers may not be clinically significant [21]. Currently, sampling via fine-needle aspiration (FNA) is used to guide the therapeutic management of patients with thyroid nodules. Indeed, the use of FNA can reduce unnecessary thyroid surgery by $25 \%$ [23]. Nonetheless, the prevalence of FNA samples with indeterminate and non-diagnostic cytology remains high at approximately 30\% [23]. Furthermore, because the prognosis of most cases of TC is so good that controlled studies are almost impossible, there are still many unanswered questions regarding the best form of treatment [21]. Consequently, there is a need to focus more efforts on the effective diagnosis, control, and treatment of TC. In particular, understanding the underlying molecular mechanisms of TC will aid the development of effective individualized treatments.

AITD has an estimated prevalence of 5\%, making it one of the most widespread autoimmune diseases [14]. The criteria used for diagnosis of AITD include circulating concentrations of autoantibodies and thyroid stimulating hormone, as well as clinical and biochemical features, such as classical features on thyroid ultrasounds. By contrast, FNA cytology and radioactive iodine absorption are rarely used as diagnostic indicators of AITD [12]. Treatment of the hypothyroidism that arises as a result of AITD includes daily consumption of synthetic L-T4 (1.5-1.7 $\mu \mathrm{g} / \mathrm{kg}$ body weight/day) [12]. However, there remain major challenges in providing effective healthcare for AITD patients, such as accurate diagnosis, choosing the right treatment, accurately predicting therapeutic responses, and providing appropriate personalized treatments [24]. Thus, it is important to explore new potential biomarkers and therapeutic targets of AITD.

\subsection{Circular RNAs}

\subsubsection{Structure, classification, and biogenesis of circRNAs}

Based on their patterns of biogenesis from genomic regions, circular RNAs (circRNAs) can be divided into four categories: exonic circRNAs, circular intronic RNAs, exon-intron circRNAs, and intergenic circRNAs $[25,26]$. There are many hypotheses for the mechanism of circRNA formation, among which the lariat-driven circularization and intron-pairing-driven circularization mechanisms are the most widely accepted (Figure 1) [27]. Research into the formation of circRNAs has had a positive catalytic effect on the exploration of their functions; however, many proposed mechanisms require further analysis and verification. Unlike linear RNAs, which are terminated with $5^{\prime}$ caps and $3^{\prime}$ tails, circRNAs are characterized by covalently closed loop structures that lack $5^{\prime}$ to $3^{\prime}$ polarity and polyadenylated tails. This intrinsic characteristic has led to a general underestimation of the existence of circular RNAs in previous polyadenylated transcriptome analyses [28]. However, in recent years, RNA sequencing technologies and bioinformatics approaches have identified a large number of circRNAs in humans [29-31], mice [29, 30], nematodes [29, 32], zebrafish [33], fruit flies [34], protists [35], and plants [35]. Furthermore, these technologies have enabled characterization of the abundance and diversity of circRNAs, as well as their dynamic expression patterns in various developmental stages and physiological conditions.

Many circRNAs are expressed in a tissue-specific manner. For example, circRmst and circKlhl2 are expressed at high levels in the mouse brain but are not found in the liver or lung [36], and circ-MBL expression in the Drosophila ovary is much lower than that in the head [37]. The expression levels of some circRNAs also change at different ages or developmental stages. For example, one study found that the levels of multiple circRNAs, including MM9_ circ_004501 and MM9_circ_013636, were higher in the brains of 22-month-old rats than in those of 1-year-old rats [29].

\subsubsection{Functions of circRNAs}

To date, circRNAs have been reported to function as competing endogenous RNAs or "miRNA sponges" [26, 38, 39, 40], mediators of RNA/protein interactions [39], and scaffolds in the assembly of protein complexes [42]. In addition, circRNAs can be translated into functional proteins, sequester proteins from their native subcellular localization [43], 
modulate the expression of parental genes [38, 44-46], regulate alternative splicing [41], and modulate the stability of mRNAs [26].

The ability of circRNAs to function as miRNA sponges has been studied in most detail. The presence of miRNA binding sites or response elements in some circRNAs enables them to regulate the function of miRNAs by acting as competitive endogenous RNAs (ceRNAs) or "miRNA sponges" [26, 40, 46, 47]. Competitive binding of miRNAs to ceRNAs reduces the expression levels of the free miRNAs and inhibits their ability to affect gene expression at the transcriptional or post-transcriptional level [25, 48, 49]. However, the biological significance of the classic role of ceRNAs as miRNA sponges is being increasingly debated, and a recent study demonstrated that most circRNAs do not function in the same way as "real" miRNA sponges [50].

The protein interactions of a number of circRNAs, such as circ-Foxo3 [39, 42] and circ008274 [51], have been studied extensively in disease states, especially tumors. Ectopic expression of circ-Foxo3 inhibits cell cycle progression by binding to the cell cycle proteins cyclin-dependent kinase 2 and cyclindependent kinase inhibitor 1 (p21) [39].

Moreover, a number of endogenous circRNAs can be translated into proteins [52-54], which calls into doubt the notion that they function as non-coding RNAs exclusively. Other studies have shown that synthesized circRNAs containing multiple FLAGcoding sequences can also be translated into proteins in the absence of a particular element for internal ribosome entry, through a mechanism similar to rolling circle amplification [55].

CircRNAs can also regulate gene transcription. For example, circ-EIF3J and circ-PAIP2 can combine with the U1 small nuclear ribonucleoprotein to interact with RNA polymerase II and enhance the expression of their parental genes in HeLa and HEK2393 cells [44].

The enormous number of circRNAs identified to date suggests that they may have complex and diverse functions. In terms of characterizing the mechanisms underlying tumor formation and development, the roles of circRNAs as miRNA sponges have been studied in most detail, followed by their interactions with proteins. However, the relationships between cancer and the abilities of circRNAs to be translated into proteins, modulate the stability of mRNAs and the splicing of pre-RNAs, and regulate parental gene transcription have not been studied in detail. These potential new roles of circRNAs in tumor development require further investigation.

\subsubsection{CircRNAs as tumor markers}

Recently, genome-wide analyses have shown that various circRNAs play a role in a number of pathological processes, including the development of cancer, through transcriptional and posttranscriptional regulatory mechanisms [56-58]. The identification of differences in gene expression levels between tumor and normal samples is an important component of cancer biology research. Analyses of a variety of tumor cells and corresponding normal cells have revealed abnormal circRNA expression levels in a variety of cancers [58]. In addition, other studies have shown that circRNAs participate in the initiation and progression of tumors, and can be used as molecular markers of the activation of signal transduction pathways related to cancer development [59-63].

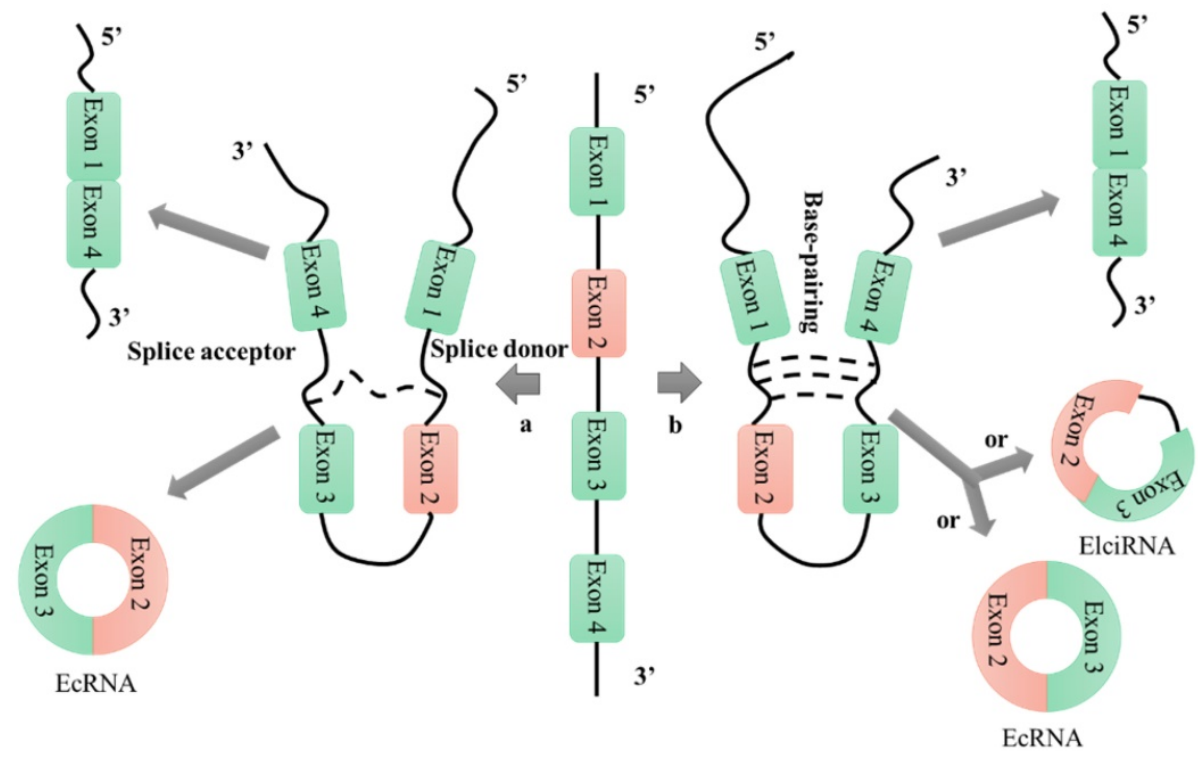

Figure 1. CircRNA formation mechanisms. a: lariat-driven circularization; b: intron-pairing-driven circularization. 
Although a large number of studies have examined the use of proteins and miRNAs as biomarkers of cancer [64, 65], circRNAs have a number of unique advantages. The main advantages of circRNAs as cancer biomarkers include their ability to be detected via RT-PCR analyses of minimally invasive blood, urine, or saliva samples [43, 66, 67], as well as their high circulating stability. Indeed, circRNAs are unusually stable RNA molecules, presumably because they lack an open end and are therefore able to avoid conventional RNA degradation pathways [58]. Notably, circRNAs play an important role in regulating the $\mathrm{Wnt} / \beta$-catenin pathway [43, 68, 69], an important signaling mechanism involved in tumor progression [70]. It is possible that targeted inhibition of circRNAs could block the Wnt/ $\beta$-catenin pathway to inhibit tumor growth and development.

\section{CircRNAs and TC}

In recent years, several studies have shown that a number of circRNAs are abnormally expressed in tumor tissues and are related to cancer progression [71]. Differential expression of these circRNAs can lead to changes in both biological processes and the flow of genetic information. CircRNAs play roles in TC signaling pathways and the invasion and migration of TC cells. In addition, the expression levels of circRNAs can be used for diagnosis of TC and monitoring the response to therapy. Table $\mathbf{1}$ summarizes the changes in expression levels (i.e., up-regulation or down-regulation) and regulatory functions of circRNAs in TC reported in recent studies. However, due to the high proportion of PTC among all thyroid cancers (about 80\%) [11], recent studies on circRNA and TC have largely focused on PTC.

Table 1. Dysregulated circRNAs in thyroid cancer (TC)

\begin{tabular}{|c|c|c|c|c|c|c|}
\hline Name & Dysregulation & $\begin{array}{l}\text { Sponge } \\
\text { target }\end{array}$ & $\begin{array}{l}\text { Downstream } \\
\text { target }\end{array}$ & Function & Material Source & Reference \\
\hline circFOXM1 & up-regulated & miR-1179 & HMGB1 & promotes PTC development & 78 PTC and paired non-tumorous tissue samples & [77] \\
\hline \multirow[t]{2}{*}{ circ_0058124 } & \multirow[t]{2}{*}{ up-regulated } & miR-218-5p & $\begin{array}{l}\text { NOTCH3/ } \\
\text { GATAD2A }\end{array}$ & \multirow{2}{*}{$\begin{array}{l}\text { promotes PTC proliferation, invasion, } \\
\text { and metastasis and associates with the } \\
\text { malignant features and poor outcomes }\end{array}$} & 92 PTC and paired non-tumorous tissue samples & [56] \\
\hline & & miR-940 & MAPK1 & & 51 TC and paired non-tumorous tissue samples & [81] \\
\hline circ_0039411 & up-regulated & $\begin{array}{l}\operatorname{miR}-1179 \\
\operatorname{miR}-1205\end{array}$ & $\begin{array}{l}\text { ABCA9 } \\
\text { MTA1 }\end{array}$ & $\begin{array}{l}\text { promotes PTC growth, migration, and } \\
\text { invasion and suppresses cell apoptosis }\end{array}$ & 46 patients with PTC & [83] \\
\hline circPRMT5 & up-regulated & $\operatorname{miR}-30 \mathrm{c}$ & E2F3 & $\begin{array}{l}\text { promotes advanced stage and lymph } \\
\text { node metastasis }\end{array}$ & 55 PTC and paired non-tumorous tissue samples & [80] \\
\hline circ_0011385 & up-regulated & $\operatorname{miR}-361-3 p$ & - & $\begin{array}{l}\text { promotes TC proliferation, migration } \\
\text { and invasion and suppresses cell cycle } \\
\text { arrest and apoptosis }\end{array}$ & $15 \mathrm{TC}$ and paired non-tumorous tissue samples & [82] \\
\hline circZFR & up-regulated & miR-1261 & C8orf4 & $\begin{array}{l}\text { promotes TC proliferation, migration } \\
\text { and invasion }\end{array}$ & 41 PTC and paired non-tumorous tissue samples & [75] \\
\hline circ-ITCH & $\begin{array}{l}\text { down- } \\
\text { regulated }\end{array}$ & $\operatorname{miR}-22-3 p$ & $\begin{array}{l}\text { CBL/Wnt/ } \\
\beta \text {-catenin }\end{array}$ & $\begin{array}{l}\text { suppresses PTC proliferation and } \\
\text { invasion and enhances apoptosis }\end{array}$ & $\begin{array}{l}37 \text { PTC and } 14 \text { matched non-tumor tissues (all PTC } \\
\text { patients did not receive any treatment before surgical } \\
\text { removal of the tumor) }\end{array}$ & [86] \\
\hline circ_0067934 & up-regulated & miR-1304 & CXCR1 & $\begin{array}{l}\text { suppresses TC apoptosis and promotes } \\
\text { proliferation, migration, and invasion }\end{array}$ & $\begin{array}{l}50 \mathrm{TC} \text { and paired non-tumorous tissue samples (all TC } \\
\text { patients did not receive any treatment before surgical } \\
\text { removal of the tumor) }\end{array}$ & [85] \\
\hline circ_0025033 & up-regulated & $\begin{array}{l}\operatorname{miR}-1231 \\
\operatorname{miR}-1304\end{array}$ & - & $\begin{array}{l}\text { promotes PTC proliferation, migration } \\
\text { and invasion and suppresses cell } \\
\text { apoptosis }\end{array}$ & $\begin{array}{l}8 \text { PTC and paired non-tumorous tissue samples (all } \\
\text { PTC patients did not receive any treatment before } \\
\text { surgical removal of the tumor) }\end{array}$ & [84] \\
\hline circTP53 & up-regulated & miR-1233-3p & MDM2/p53 & promotes cancer cell proliferation & $\begin{array}{l}25 \text { PTC and paired non-tumorous tissue samples ( } 23 \\
\text { cases were differentiated thyroid carcinoma, and } 2 \\
\text { cases were poorly differentiated tumors) }\end{array}$ & [76] \\
\hline circRAPGEF5 & up-regulated & miR-198 & FGFR1 & $\begin{array}{l}\text { promotes PTC proliferation and } \\
\text { migration, a potential biomarker and } \\
\text { therapeutic target }\end{array}$ & $\begin{array}{l}30 \text { PTC and paired non-tumorous tissue samples (all } \\
\text { PTC patients did not receive any treatment before } \\
\text { surgical removal of the tumor) }\end{array}$ & [114] \\
\hline circNUP214 & up-regulated & $\operatorname{miR}-145$ & ZEB2 & $\begin{array}{l}\text { promotes PTC proliferation, migration, } \\
\text { and invasion }\end{array}$ & 30 PTC and paired non-tumorous tissue samples & [79] \\
\hline circ_0004458 & up-regulated & miR-885-5p & RAC1 & $\begin{array}{l}\text { promotes PTC progression, a potential } \\
\text { therapeutic target and biomarker }\end{array}$ & 48 PTC and paired non-tumorous tissue samples & [61] \\
\hline circLDLR & up-regulated & miR-195-5p & LIPH & $\begin{array}{l}\text { promotes PTC proliferation, migration, } \\
\text { and invasion }\end{array}$ & 60 PTC and paired non-tumorous tissue samples & [78] \\
\hline $\operatorname{circBACH} 2$ & up-regulated & miR-139-5p & $\mathrm{LMO} 4$ & a tumor biomarker of PTC & 40 PTC and paired non-tumorous tissue samples & [62] \\
\hline circ102171 & up-regulated & CTNNBIP1/W & Vnt/ $\beta$-catenin & promotes `PTC progression & 47 PTC and paired non-tumorous tissue samples & [69] \\
\hline $\operatorname{circ0008274}$ & up-regulated & $\mathrm{AMPK} / \mathrm{mTOR}$ & & $\begin{array}{l}\text { promotes PTC proliferation and } \\
\text { invasion, a novel therapeutic candidate } \\
\text { target }\end{array}$ & $\begin{array}{l}\text { Primary PTC tissues and paired non-tumorous tissue } \\
\text { samples }\end{array}$ & [51] \\
\hline $\operatorname{circ0000285}$ & up-regulated & miR-599 & -- & $\begin{array}{l}\text { promotes TC metastatic abilities, a } \\
\text { potential therapeutic target }\end{array}$ & $\begin{array}{l}\text { TC and paired non-tumorous tissue samples (all PTC } \\
\text { patients did not receive any treatment before surgical } \\
\text { removal of the tumor) }\end{array}$ & [103] \\
\hline $\operatorname{circFNDC3B}$ & up-regulated & miR-1178 & TLR4 & a novel biomarker & $\begin{array}{l}\text { PTC and paired non-tumorous tissue samples (all PTC } \\
\text { patients did not receive any treatment before surgical } \\
\text { removal of the tumor). For exosome purification, serum } \\
\text { samples were collected from these } 42 \text { cases of PTC and } \\
40 \text { cases of healthy donors. }\end{array}$ & [63] \\
\hline
\end{tabular}


The link between inflammation and cancer is well known. Over the past few decades, many studies have shown that AITD and TC (mainly PTC) can occur simultaneously, and that the increase in TC incidence coincides with the increase in AITD registration. However, the mechanism responsible for the link between AITD and TC is still unclear [72]. Therefore, in this review, TC and AITD are described together in the hope that future studies will uncover the link between these two diseases and their relationships to circRNA levels.

\subsection{Expression levels of circRNAs in TC}

Recent studies have demonstrated that a number of circRNAs are expressed at abnormal levels in TC [71], including PTC. A microarray analysis of six PTC tumors, six matched normal control samples, and 18 thyroid samples from six benign thyroid lesions revealed that circRNA imbalance may play a role in the pathogenesis of PTC, and identified some key circRNAs as candidate biomarkers of the disease [73]. Another study using a high-throughput sequencing analysis found altered levels of exosomal circRNAs in the serum of patients with PTC [74]. Specifically, compared with those from the control group (patients diagnosed with benign goiter), serum-derived exosomes from PTC patients contained three up-regulated circRNAs and 19 down-regulated circRNAs. These differentially expressed circRNAs were related to 16 signaling pathways [74]. Abnormal expression of circRNAs in PTC may result in changes in tissue function and metabolism, thereby representing a molecular mechanism underlying the development of PTC.

\subsection{The emerging roles of circRNAs in TC proliferation, invasion, and progression}

Abnormal cell proliferation, migration, and invasion are the main characteristics of TC. Studies performed in recent years have shown that circRNAs may be associated with these abnormal characteristics of TC cells.

Indeed, several circRNAs are closely associated with TC cell proliferation. Most of the circRNAs that promote thyroid cell proliferation exert their roles by functioning as miRNA sponges [75-79]. The expression level of circZFR is correlated with the clinical severity of PTC [75]. CircZFR reportedly promotes the expression of C8orf4 by acting as a ceRNA that targets miR-1261, thereby enhancing the proliferation, migration, and invasion of PTC cells. Moreover, Ye et al. showed recently that circFOXM1 promotes the progression of PTC by modulating the miR-1179/HMGB1 pathway [77]. Another study found that circ-LDLR regulates LIPH expression by acting as a miRNA sponge targeting miR-195-5p, thereby promoting PTC progression [78]. Li et al. showed that circNUP214 may play an oncogenic role in PTC by acting as a sponge for miR-145, leading to up-regulation of its target zinc finger E-box binding homeobox 2 [79]. In addition, Ma et al. reported that direct targeting of miR-1233-3p by circTP53 increases the level of the MDM2 mRNA and reduces p53 expression in TC cells, thereby promoting cell proliferation [76]. In addition to these circRNAs that act as miRNA sponges, Bi et al. found that circ-102171 functions as an oncogene in PTC by interacting directly with the CTNNBIP1 protein. This interaction promotes cancer development by activating the $\beta$-catenin pathway [70].

In addition to the stimulatory effects of specific circRNAs on TC cell proliferation and migration, several circRNAs promote the metastasis or lymph node metastasis of these cells. Xue's group demonstrated that the level of circPRMT5 is positively correlated with advanced stage PTC and lymph node metastasis [80]. Further investigation revealed that circPRMT5 functions as a competing endogenous RNA for miR-30c. The inhibition of miR-30c by circPRMT5 promotes the expression of E2F3, a crucial oncogene in several tumors. Sun et al. demonstrated that circ_0058124 might play a pro-metastatic role in TC by regulating the miR-940/MAPK1 axis [81]. In addition, Yao et al. found that circ_0058124 acts as an oncogenic driver that promotes PTC cell proliferation, tumorigenicity, tumor invasion, and metastasis. Specifically, circ_0058124 functions as a competing endogenous RNA to modulate the expression levels of miRNA-218-5p and its target gene NUMB, leading to repression of the NOTCH3/GATAD2A signaling axis [57].

A group of circRNAs can inhibit the apoptosis of cancer cells. Xia at el. found that hsa_circ_0011385 suppresses the cell cycle arrest and apoptosis of PTC cells in vitro by targeting miR-361-3p via a miRNA sponge process [82]. Similarly, Yang et al. demonstrated that hsa_circ_0039411 represses the apoptosis of PTC cells by functioning as a sponge for miR-1179 and miR-1205. Inhibition of miR-1179 and miR-1205 increases the expression levels of the mRNAs encoding ATP-binding cassette transporter A9 and metastasis-associated 1, respectively, at the post-transcriptional level [83]. Furthermore, circ 0025033 promotes PTC cell proliferation, migration, and invasion, and inhibits cell apoptosis by acting as a sponge for miR-1231 and miR-1304 [84]. In addition to circRNAs that act as miRNA sponges, circ_0067934 interacts with proteins directly to promote TC cell proliferation, migration, and invasion, and inhibit cell 


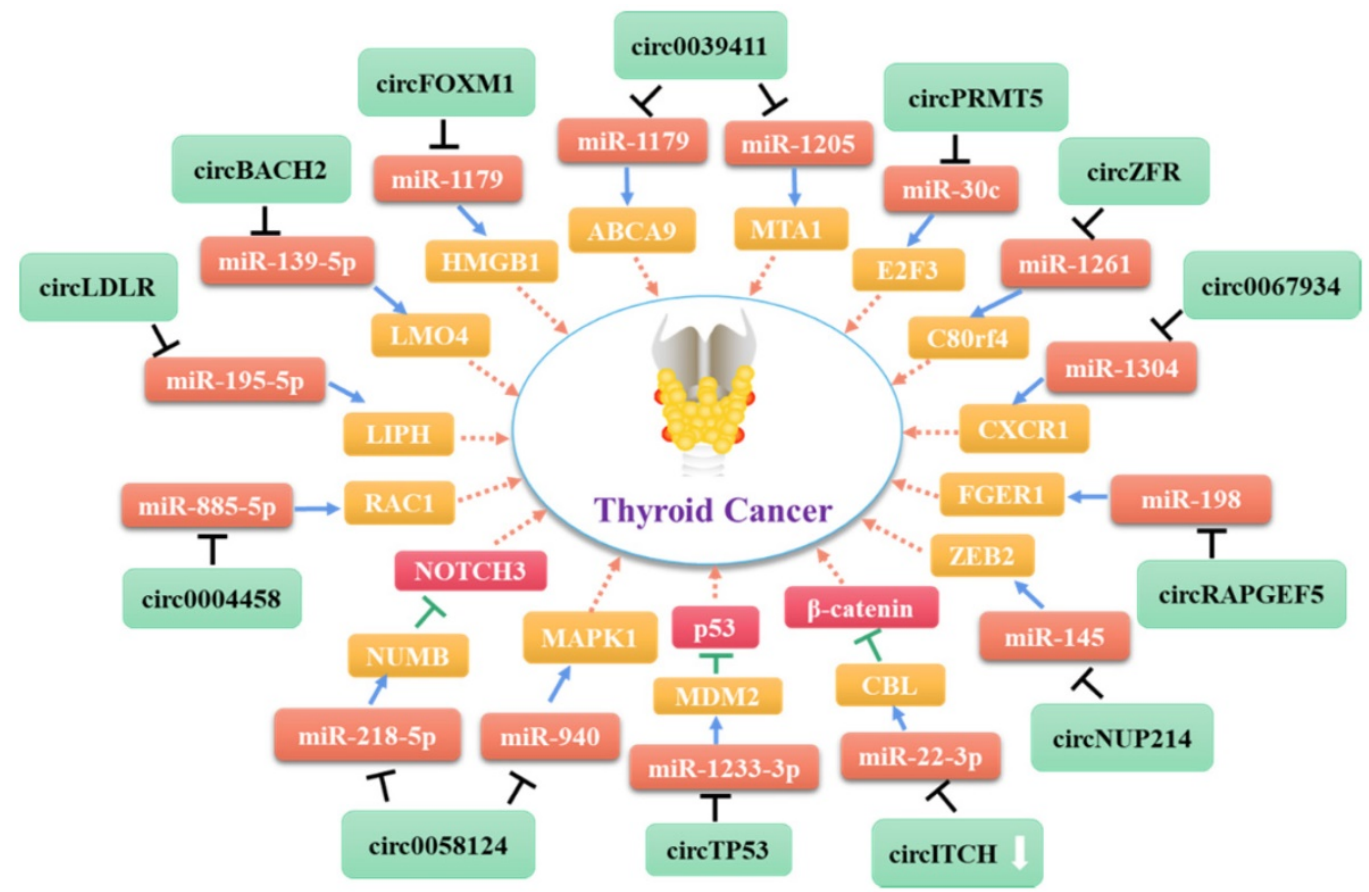

Figure 2. Molecular mechanism network of circRNA in thyroid cancer (CircRNAs serve as miRNA sponges).

apoptosis by promoting the EMT and PI3K/AKT signaling pathways [85].

To date, only one circRNA, namely circ-ITCH, has been found to be down-regulated in TC [86]. Functional tests showed that overexpression of circ-ITCH inhibits the proliferation and invasion of PTC cells, and promotes cell apoptosis by binding to miR-22-3p and inhibiting its ability to up-regulate the expression of CBL (Cbl proto-oncogene), an E3 ligase targeting nuclear $\beta$-catenin. The resulting increase in CBL expression inhibits activation of the $W n t / \beta-$ catenin pathway, thereby inhibiting the progression of PTC.

As shown in Table 1, most of the circRNAs related to TC progression are up-regulated in tumor cells and exert their roles by functioning as miRNA sponges [75, 82, 86] (Figure 2). Some other circRNAs act directly on proteins or bind to miRNAs to control cancer progression (Figure 3). As mentioned above (see section 1.2.1), some circRNAs contain one or more miRNA binding sites [86] that enable them to sequester miRNAs and inhibit their ability to regulate the expression of their downstream target genes [87, 88]. Indeed, circRNAs have been used widely as miRNA sponges to regulate target genes in cancer research. Using the GEO and TCGA databases, Liu et al. integrated circRNA, miRNA, and mRNA data from PTC tissues and non-tumor tissues to construct a circRNA-miRNA-mRNA regulatory network. This approach revealed that circRNAs regulate TC cell proliferation, apoptosis, and invasion by targeting miRNAs [88].
Overall, increasing efforts have been made to explore the mechanisms by which circRNAs regulate TC progression; such investigations have provided new insights into the pathogenesis of the disease.

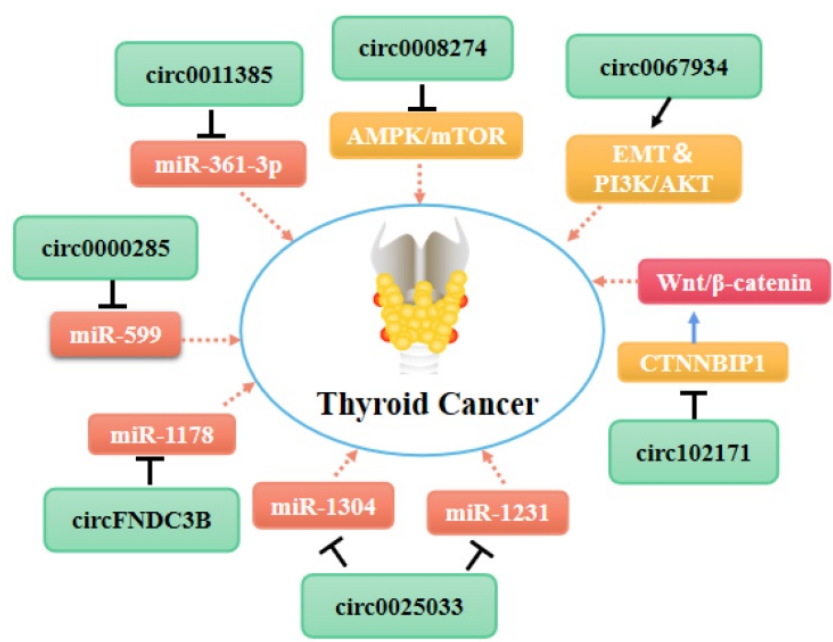

Figure 3. Molecular mechanism network of circRNA in thyroid cancer (CircRNAs bind with proteins or miRNAs).

\subsection{CircRNAs as biomarkers for the diagnosis and treatment of TC}

\subsubsection{CircRNAs as biomarkers for TC diagnosis}

The incidence of TC, especially that of PTC, has increased rapidly over recent decades, and only $59 \%$ of patients with progressed TC have a 5-year overall survival rate [21]. At present, diagnosis of thyroid 
tumors involves the use of ultrasound and radionuclide scanning, as well as various other procedures [89]. The initial risk estimate, typically derived from ultrasound and sometimes cytology reports, should determine the need for treatment and the type, frequency, and length of subsequent follow-ups [90]. However, accurate identification of TC from indeterminate cytological samples is challenging, and ultrasonographic features can be misleading in these types of tumors [91].

Traditionally, blood biomarkers have been widely used to diagnose cancer, but their low sensitivity and specificity has limited their applications for early diagnosis and prognosis [92]. Therefore, biomarkers with high specificity and sensitivity are urgently needed. Recently, circRNAs were reported to be ideal liquid biopsy biomarkers. Additionally, circRNA expression patterns show significant differences between TC patients and healthy controls, and many circRNAs show tissue-specific and developmental stage-specific expression patterns and play critical roles in cancer-related biological processes [59]. In view of their molecular stability, rich diversity, and tissue specificity, circRNAs in body fluids have potential as novel biomarkers for the monitoring of the development and progression of TC [93].

Shi et al. [94] found that the expression levels of two circRNAs (circRAPGEF5 and hsa_circ_0058124) were significantly higher in thyroid samples from PTC patients than in those from healthy controls or in benign thyroid nodules. In addition, the combination of the expression levels of these two circRNAs was independently correlated with the occurrence of PTC, indicating that they could be used as new diagnostic biomarkers of the disease. Other studies have shown that circMAN1A2 [95], hsa_circ_0102272 [96], hsa_ circ_0124055 [97], hsa_circ_0101622 [97], hsa_circ_ 0137287 [98], and circ_0006156 [99] play important roles in regulating the proliferation, migration, and apoptosis of TC cells, and could also be used as biomarkers of PTC. Despite these findings, research into the potential use of circRNAs as diagnostic biomarkers of $\mathrm{TC}$ is still at an early stage. More detailed investigations are certainly required before circRNAs can be considered as biomarkers for clinical application. Nonetheless, the stability of circRNAs in exosomes and plasma suggests that they may represent a convenient method of diagnosing TC. In addition, the diversity and tissue-specific expression of circRNAs give them advantages over more traditional biomarkers, which tend to have low organ specificity.

\subsubsection{CircRNAs as therapeutic targets for TC}

TC is a malignant tumor with an increasing incidence [17, 18]. However, the current clinical approach to treatment of TC, which involves surgery and/or radiotherapy or chemotherapy, results in low recovery rates and high recurrence rates; hence it is not sufficiently effective [100]. Moreover, radiotherapy can produce obvious side effects, while surgery can affect the normal physiological structure and function of patients [101, 102]. Therefore, there is an urgent need to develop a new treatment strategy for thyroid tumors.

Targeted regulation of specific circRNAs may be a new therapeutic approach for thyroid tumor patients. Indeed, a number of studies have shown that various circRNAs, including circFOXM1 [77], hsa circ_0058124 [57, 81], and hsa_circ_0039411 [83], play a role in the growth, migration, invasion, and development of thyroid tumors. Recently, Zhou et al. found that high has-circ-0008274 expression increases PTC cell proliferation and invasion [52], and the has-circ-0008274/AMPK/mTOR axis may be a novel therapeutic candidate target for PTC. Other circRNAs, including circ-0004458 [62], circ-BACH2 [63], circFNDC3B [64], circ-0000285 [103], play unique roles in regulating TC progression. These circRNAs could represent new therapeutic targets for thyroid tumors.

Drug resistance is a prominent problem associated with the treatment of thyroid tumors, especially anaplastic thyroid carcinoma (ATC) [104]. Due to the undifferentiated phenotype and aggressive nature of ATC, resistance to conventional treatments such as radiotherapy and chemotherapy, including resistance to cisplatin, is observed often [105, 106]. Therefore, patients with ATC tend to have a very poor prognosis. CircEIF6 can promote cisplatin-induced autophagy by regulating the hsa_miR-144-3p/TGF-a axis, thereby inhibiting cell apoptosis and enhancing the resistance of PTC and ATC cells to cisplatin [104]. Therefore, it may be possible to enhance the sensitivity of thyroid tumor patients to cisplatin and to enhance the effect of chemotherapy by knocking out circEIF6.

To date, only a few studies have investigated the use of circRNAs as therapeutic targets for thyroid tumors, and further research is clearly needed.

\section{CircRNAs and AITD}

Although current understanding of the pathogenesis of HT remains very limited, it does depend on a number of factors, including genetic susceptibility and environmental and immune factors [107-110]. Some investigations have shown that circRNAs play a role in the occurrence and development of HT. For instance, the expression level 
of hsa_circ_0089172 is increased markedly in HT patients and is inversely correlated with reduced levels of miR-125a-3p. Thus, hsa_circ_0089172 could be regarded as a potential diagnostic biomarker of HT and may play a crucial role in the pathogenesis of HT by acting as a miRNA sponge toward miR-125a-3p [111]. However, research in this area is still in its infancy and only a few relevant studies have been published. Generating a complete profile of circRNA expression in HT patients would aid the identification of new biomarkers and the development of novel strategies for HT diagnosis and treatment.

CircRNAs may also play a role in the development of GD, another AITD. Sun et al. isolated serum exosomes from five primary GD patients and five healthy controls, and confirmed that hsa_circRNA_000102 was up-regulated significantly in those from GD patients [112]. Moreover, analysis of the circRNA/miRNA/mRNA interaction network revealed the potential miRNA targets of hsa circRNA_000102 and their related downstream target genes [112]. This novel investigation provides a feasible framework for the identification and validation of circRNA biomarkers.

Thyroid-associated ophthalmopathy (TAO) is one of the most common clinical manifestations of GD patients; however, the mechanism underlying TAO is not fully understood. Wu et al. used high-throughput RNA sequencing of orbital fat/connective tissue samples to identify mRNAs and circRNAs that were differentially expressed between the control group and patients with TAO [113]. In addition, $\mathrm{Wu}$ et al. revealed the roles of the differentially expressed circRNAs in TAO by circRNA-mRNA co-expression and circRNA/miRNA interaction analyses. The results of these analyses suggested that differentially expressed circRNAs may be involved in the pathogenesis of TAO, and that the circRNA_14940CCND1-Wnt signaling pathway may be an important regulatory axis [113].

Overall, only a few studies have examined the role of circRNAs in the development of AITD, and research in the field is still in its infancy. The functions of the majority of circRNAs remain unclear, and their relationship to the occurrence and development of AITD require further investigation and clarification. The identification of circRNAs that are differentially expressed in AITD may provide insights into the pathogenesis of the disease.

\section{Conclusions and perspective}

TC and AITD are common thyroid diseases with high incidences. In recent years, the roles of circRNAs in diseases, including various cancers, have been investigated vigorously. In this review, we have systematically discussed the roles of circRNAs in TC and AITD, and described their potential use for the diagnosis and treatment of these diseases.

Accumulating evidence suggests that control of the transcriptome plays an important role in tumorigenesis and the progression of TC. Notably, circRNAs play a critical role in transcriptional and post-transcriptional regulation during tumorigenesis and TC development. As a relatively new focus of transcriptomic regulation, the molecular stability, substantial diversity, and tissue specificity of circRNAs have led to investigations of their use as diagnostic biomarkers of disease. Indeed, studies of circRNAs have deepened our understanding of the complex regulated network of non-coding RNAs. One of the main advantages of circRNAs as biomarkers is their high circulating stability and ability to be detected via RT-PCR analyses of minimally invasive blood, urine, or saliva samples. In addition, circRNAs might be useful therapeutic agents and/or targets. The use of circularized miRNA sponges in cells is a new candidate for RNA-based cancer treatments. Furthermore, examining the differences between circRNA levels in tumors and normal tissues is a topic for future clinical research, although large clinical datasets will need to be examined. However, at present, most research is focused on the relationship between circRNA levels and PTC, while other subtypes of TC are largely ignored. Although these subtypes do not account for most TC, they still have clinical research value.

In addition to TC, circRNAs play an important role in the pathogenesis of AITD, although research into this area is still in its infancy. A number of circRNAs are differentially expressed in various AITDs, but the mechanisms of action of these circRNAs are still unclear and require further verification. Examining the abnormal expression patterns of circRNAs during the development of AITDs will improve our understanding of the molecular basis of these disorders, and will provide a theoretical basis for the use of circRNAs as diagnostic markers and possible therapeutic targets of AITD.

Although many studies have shown that AITD i $\mathrm{s}$ closely related to $\mathrm{TC}$, the interaction and association in circRNA has not been studied. Future studies will be necessary to understand the connections between thyroid autoimmunity and cancer, to allow the design of tailored therapies for TC patients with AITD.

The potential use of circRNAs for clinical applications faces a number of challenges. First, the molecular mechanisms underlying the physiological and pathological effects of circRNAs remain largely unknown. Before they can be considered for use as biomarkers or therapeutic targets of TC and AITD, 
additional research is required to define their precise functions and mechanisms of action. Second, the naming standards for circRNAs are not uniform. Thus, numerous authoritative studies cannot be unified and generalized easily. Third, the potential use of circRNAs as therapeutic targets in clinical practice is currently at the stage of inference and theory; potential problems related to side effects, drug resistance, therapeutic effects, and administration mode remain unknown.

In conclusion, through the development of techniques to identify and screen novel circRNAs, as well as the improvement of online databases, it is possible that circRNAs will one day be used widely for the diagnosis, monitoring, and treatment of thyroid diseases.

\section{Abbreviations}

AITD: autoimmune thyroid disease; ATC: anaplastic thyroid carcinoma; CBL: $\mathrm{Cbl}$ protooncogene; ceRNA: competitive endogenous RNA; circRNA: circular RNA; FNA: fine-needle aspiration; GD: Graves' disease; HT: Hashimoto's thyroiditis; miRNA: microRNA; PTC: papillary thyroid carcinoma; p21: cyclin-dependent kinase inhibitor 1; TAO: thyroid-associated ophthalmopathy; TC: thyroid cancer.

\section{Acknowledgements}

This work was supported by the National Natural Science Foundation of China (22006084); Major Research Program of the National Natural Science Foundation of China (91849209); the Qingdao Applied Basic Research Project (19-6-2-49-cg) and the People's Livelihood Science and Technology Project of Qingdao (18-6-1-63-nsh); and Hubei Key Laboratory of Environmental and Health Effects of Persistent Toxic Substances (PTS2019-05).

\section{Competing Interests}

The authors have declared that no competing interest exists.

\section{References}

1. Frazell EL, Foote FW, Jr. The natural history of thyroid cancer; a review of 301 cases. J Clin Endocrinol Metab. 1949; 9: 1023-30.

2. Nikiforov $\mathrm{YE}$, Nikiforova MN. Molecular genetics and diagnosis of thyroid cancer. Nat Rev Endocrinol. 2011; 7: 569-80.

3. Kondo T, Ezzat S, Asa SL. Pathogenetic mechanisms in thyroid follicular-cell neoplasia. Nat Rev Cancer. 2006; 6: 292-306.

4. Burn JI, Taylor SF. Natural history of thyroid carcinoma. A study of 152 treated patients. Br Med J. 1962; 2: 1218-23.

5. Takano T. Natural history of thyroid cancer. Endocr J. 2017; 64: 237-44.

6. Ahn HS, Kim HJ, Welch HG. Korea's thyroid-cancer "epidemic"--screening and overdiagnosis. N Engl J Med. 2014; 371: 1765-7.

7. Ahn HS, Welch HG. South Korea's Thyroid-Cancer "Epidemic"--Turning the Tide. N Engl J Med. 2015; 373: 2389-90.

8. Wada N, Duh QY, Sugino K, et al. Lymph node metastasis from 259 papillary thyroid microcarcinomas: frequency, pattern of occurrence and recurrence, and optimal strategy for neck dissection. Ann Surg. 2003; 237: 399-407.
9. Ito $\mathrm{Y}$, Miyauchi A, Kihara M, et al. Patient age is significantly related to the progression of papillary microcarcinoma of the thyroid under observation. Thyroid. 2014; 24: 27-34.

10. Suzuki S. Childhood and Adolescent Thyroid Cancer in Fukushima after the Fukushima Daiichi Nuclear Power Plant Accident: 5 Years On. Clin Oncol (R Coll Radiol). 2016; 28: 263-71.

11. Thun MJ, Linet MS, Cerhan JR, et al. Cancer epidemiology and prevention 4th ed. New York, USA: Oxford University; 2018.

12. Caturegli P, De Remigis A, Rose NR. Hashimoto thyroiditis: clinical and diagnostic criteria. Autoimmun Rev. 2014; 13: 391-7.

13. Jacobson DL, Gange SJ, Rose NR, et al. Epidemiology and estimated population burden of selected autoimmune diseases in the United States. Clin Immunol Immunopathol. 1997; 84: 223-43.

14. Antonelli A, Ferrari SM, Corrado A, et al. Autoimmune thyroid disorders. Autoimmun Rev. 2015; 14: 174-80.

15. Hiromatsu $Y$, Satoh H, Amino N. Hashimoto's thyroiditis: history and future outlook. Hormones (Athens, Greece). 2013; 12: 12-8.

16. Kassotis CD, Herkert NJ, Hammel SC, et al. Thyroid Receptor Antagonism of Chemicals Extracted from Personal Silicone Wristbands within a Papillary Thyroid Cancer Pilot Study. Environ Sci Technol. 2020; 54: 15296-312.

17. Zhang YF, $\mathrm{Xu} \mathrm{HM}, \mathrm{Yu}$ F, et al. Comparison the sensitivity of amphibian metamorphosis assays with NF 48 stage and NF 51 stage Xenopus laevis tadpoles. Toxicol Mech Methods. 2019; 29: 421-27.

18. Lee K, Cassaro S. Cancer, Thyroid. UN California Irvine / Kaweah Delta, USA: StatPearls Publishing LLC; 2020.

19. Bray F, Ferlay J, Soerjomataram I, et al. Global cancer statistics 2018: GLOBOCAN estimates of incidence and mortality worldwide for 36 cancers in 185 countries. CA Cancer J Clin. 2018; 68: 394-424.

20. Ferlay J, Soerjomataram I, Dikshit R, et al. Cancer incidence and mortality worldwide: sources, methods and major patterns in GLOBOCAN 2012. Int J Cancer. 2015; 136: E359-86.

21. Schottenfeld D, Fraumeni JF. Cancer epidemiology and prevention. New York, USA: Oxford University; 2006.

22. Ryo UY, Arnold J, Colman M, et al. Thyroid scintigram. Sensitivity with sodium pertechnetate Tc $99 \mathrm{~m}$ and gamma camera with pinhole collimator. J Am Med Assoc. 1976; 235: 1235-8.

23. Kunavisarut T. Diagnostic biomarkers of differentiated thyroid cancer. Endocrine. 2013; 44: 616-22.

24. Yin L, Zeng C, Yao J, et al. Emerging Roles for Noncoding RNAs in Autoimmune Thyroid Disease. Endocrinology. 2020; 161.

25. Wang F, Nazarali AJ, Ji S. Circular RNAs as potential biomarkers for cancer diagnosis and therapy. Am J Cancer Res. 2016; 6: 1167-76.

26. Meng S, Zhou H, Feng Z, et al. CircRNA: functions and properties of a novel potential biomarker for cancer. Mol Cancer. 2017; 16: 94.

27. Jeck WR, Sorrentino JA, Wang K, et al. Circular RNAs are abundant, conserved, and associated with ALU repeats. RNA (New York, NY). 2013; 19: 141-57.

28. Chen LL, Yang L. Regulation of circRNA biogenesis. RNA Biol. 2015; 12: 381-8.

29. Gruner H, Cortés-López M, Cooper DA, et al. CircRNA accumulation in the aging mouse brain. Sci Rep. 2016; 6: 38907

30. Rybak-Wolf A, Stottmeister C, Glažar P, et al. Circular RNAs in the Mammalian Brain Are Highly Abundant, Conserved, and Dynamically Expressed. Mol Cell. 2015; 58: 870-85.

31. Salzman J, Chen RE, Olsen MN, et al. Cell-type specific features of circular RNA expression. PLoS Genet. 2013; 9: e1003777.

32. Ivanov A, Memczak S, Wyler E, et al. Analysis of intron sequences reveals hallmarks of circular RNA biogenesis in animals. Cell Rep. 2015; 10: 170-7.

33. Jeck WR, Sharpless NE. Detecting and characterizing circular RNAs. Nat Biotechnol. 2014; 32: 453-61.

34. Houseley JM, Garcia-Casado Z, Pascual M, et al. Noncanonical RNAs from transcripts of the Drosophila muscleblind gene. J Hered. 2006; 97: 253-60.

35. Wang PL, Bao Y, Yee MC, et al. Circular RNA is expressed across the eukaryotic tree of life. PloS one. 2014; 9: e90859.

36. You X, Vlatkovic I, Babic A, et al. Neural circular RNAs are derived from synaptic genes and regulated by development and plasticity. Nat Neurosci. 2015; 18: 603-10.

37. Westholm JO, Miura P, Olson S, et al. Genome-wide analysis of drosophila circular RNAs reveals their structural and sequence properties and age-dependent neural accumulation. Cell Rep. 2014; 9: 1966-80.

38. Xu H, Guo S, Li W, et al. The circular RNA Cdr1as, via miR-7 and its targets, regulates insulin transcription and secretion in islet cells. Sci Rep. 2015; 5: 12453.

39. Du WW, Yang W, Liu E, et al. Foxo3 circular RNA retards cell cycle progression via forming ternary complexes with p21 and CDK2. Nucleic Acids Res. 2016; 44: 2846-58.

40. Memczak S, Jens M, Elefsinioti A, et al. Circular RNAs are a large class of animal RNAs with regulatory potency. Nature. 2013; 495: 333-8.

41. Ashwal-Fluss R, Meyer M, Pamudurti NR, et al. circRNA biogenesis competes with pre-mRNA splicing. Mol Cell. 2014; 56: 55-66.

42. Du WW, Fang L, Yang W, et al. Induction of tumor apoptosis through a circular RNA enhancing Foxo3 activity. Cell Death Differ. 2017; 24: 357-70.

43. Armakola M, Higgins MJ, Figley MD, et al. Inhibition of RNA lariat debranching enzyme suppresses TDP-43 toxicity in ALS disease models. Nat Genet. 2012; 44: 1302-9. 
44. Li Z, Huang C, Bao C, et al. Exon-intron circular RNAs regulate transcription in the nucleus. Nat Struct Mol Biol. 2015; 22: 256-64.

45. Zhang $\mathrm{Y}$, Zhang $\mathrm{XO}$, Chen $\mathrm{T}$, et al. Circular intronic long noncoding RNAs. Mol Cell. 2013; 51: 792-806.

46. Bahn JH, Zhang Q, Li F, et al. The landscape of microRNA, Piwi-interacting RNA, and circular RNA in human saliva. Clin Chem. 2015; 61: 221-30.

47. Kent OA, Mendell JT. A small piece in the cancer puzzle: microRNAs as tumor suppressors and oncogenes. Oncogene. 2006; 25: 6188-96.

48. Taulli R, Loretelli C, Pandolfi PP. From pseudo-ceRNAs to circ-ceRNAs: a tale of cross-talk and competition. Nat Struct Mol Biol. 2013; 20: 541-3.

49. Hansen TB, Jensen TI, Clausen BH, et al. Natural RNA circles function as efficient microRNA sponges. Nature. 2013; 495: 384-8.

50. Militello G, Weirick T, John D, et al. Screening and validation of lncRNAs and circRNAs as miRNA sponges. Brief Bioinform. 2017; 18: 780-88.

51. Zhou GK, Zhang GY, Yuan ZN, et al. Has_circ_0008274 promotes cell proliferation and invasion involving $\mathrm{AMPK} / \mathrm{mTOR}$ signaling pathway in papillary thyroid carcinoma. Eur Rev Med Pharmacol Sci. 2018; 22: 8772-80.

52. Wesselhoeft RA, Kowalski PS, Anderson DG. Engineering circular RNA for potent and stable translation in eukaryotic cells. Nat Commun. 2018;9(1):2629.

53. Chen CY, Sarnow P. Initiation of protein synthesis by the eukaryotic translational apparatus on circular RNAs. Science. 1995;268(5209):415-7

54. Legnini I, Di Timoteo G, Rossi F, et al. Circ-ZNF609 is a circular RNA that can be translated and functions in Myogenesis. Mol Cell 2017;66(1):22-37 e29.

55. Pamudurti NR, Bartok O, Jens M, et al. Translation of CircRNAs. Mol Cell 2017;66(1):9-21 e27.

56. Yao Y, Chen X, Yang H, et al. Hsa_circ_0058124 promotes papillary thyroid cancer tumorigenesis and invasiveness through the NOTCH3/GATAD2A axis. J Exp Clin Cancer Res. 2019; 38.

57. Yin $\mathrm{Y}$, Long J, He Q, et al. Emerging roles of circRNA in formation and progression of cancer. J Cancer. 2019; 10: 5015-21.

58. Chen Y, Li C, Tan C, et al. Circular RNAs: a new frontier in the study of human diseases. J Med Genet. 2016; 53: 359-65.

59. Li P, Chen $\mathrm{S}$, Chen $\mathrm{H}$, et al. Using circular RNA as a novel type of biomarker in the screening of gastric cancer. Clin Chim Acta. 2015; 444: 132-6.

60. Chen J, Li Y, Zheng Q, et al. Circular RNA profile identifies circPVT1 as a proliferative factor and prognostic marker in gastric cancer. Cancer Lett. 2017; 388: 208-19.

61. Jin X, Wang Z, Pang W, et al. Upregulated hsa_circ_0004458 Contributes to Progression of Papillary Thyroid Carcinoma by Inhibition of miR-885-5p and Activation of RAC1. Med Sci Monit. 2018; 24: 5488-500.

62. Cai X, Zhao Z, Dong J, et al. Circular RNA circBACH2 plays a role in papillary thyroid carcinoma by sponging miR-139-5p and regulating LMO4 expression. Cell Death Dis. 2019; 10.

63. Wu G, Zhou W, Pan $X$ et al. Circular RNA Profiling Reveals Exosomal circ_0006156 as a Novel Biomarker in Papillary Thyroid Cancer. Mol Ther Nucleic Acids. 2020; 19: 1134-44.

64. Abe N, Matsumoto K, Nishihara M, et al. Rolling circle translation of circular RNA in living human cells. Sci Rep. 2015;5:16435.

65. Wang Y, Wang Z. Efficient backsplicing produces translatable circular mRNAs. RNA. 2015;21:172-9.

66. Memczak S, Papavasileiou P, Peters $\mathrm{O}$, et al. Identification and Characterization of Circular RNAs As a New Class of Putative Biomarkers in Human Blood. PloS one. 2015; 10: e0141214.

67. Lin X, Lo HC, Wong DT, et al. Noncoding RNAs in human saliva as potential disease biomarkers. Front Genet. 2015; 6: 175.

68. Huang $G$, Zhu $H$, Shi $Y$, et al. cir-ITCH plays an inhibitory role in colorectal cancer by regulating the Wnt/ $\beta$-catenin pathway. PloS one. 2015; 10: e0131225.

69. Bi W, Huang J, Nie C, et al. CircRNA circRNA_102171 promotes papillary thyroid cancer progression through modulating CTNNBIP1-dependent activation of $\beta$-catenin pathway. J Exp Clin Cancer Res. 2018; 37: 275

70. Padala RR, Karnawat R, Viswanathan SB, et al. Cancerous perturbations within the ERK, PI3K/Akt, and Wnt/ $\beta$-catenin signaling network constitutively activate inter-pathway positive feedback loops. Mol Biosyst. 2017; 13: 830-40.

71. Kristensen LS, Hansen TB, Venø MT, et al. Circular RNAs in cancer: opportunities and challenges in the field. Oncogene. 2018; 37: 555-65.

72. Ferrari SM, Fallahi $P$, Elia G, et al. Thyroid autoimmune disorders and cancer. Semin Cancer Biol. 2020; 64: 135-46.

73. Peng N, Shi L, Zhang Q, et al. Microarray profiling of circular RNAs in human papillary thyroid carcinoma. PLoS One. 2017; 12: e0170287.

74. Yang C, Wei Y, Yu L, et al. Identification of Altered Circular RNA Expression in Serum Exosomes from Patients with Papillary Thyroid Carcinoma by High-Throughput Sequencing. Med Sci Monit. 2019; 25: 2785-91.

75. Wei H, Pan L, Tao D, et al. Circular RNA circZFR contributes to papillary thyroid cancer cell proliferation and invasion by sponging miR-1261 and facilitating C8orf4 expression. Biochem Biophys Res Commun. 2018; 503: $56-61$

76. Ma W, Zhao P, Zang L, et al. CircTP53 promotes the proliferation of thyroid cancer via targeting miR-1233-3p/MDM2 axis. J Endocrinol Invest. 2020.

77. Ye M, Hou H, Shen M, et al. Circular RNA circFOXM1 Plays a Role in Papillary Thyroid Carcinoma by Sponging miR-1179 and Regulating HMGB1 Expression. Mol Ther Nucleic Acids. 2020; 19: 741-50.

78. Gui X, Li Y, Zhang X, et al. Circ_LDLR promoted the development of papillary thyroid carcinoma via regulating miR-195-5p/LIPH axis. Cancer Cell Int. 2020; 20: 241
79. Li X, Tian $\mathrm{Y}, \mathrm{Hu} \mathrm{Y}$, et al. CircNUP214 sponges miR-145 to promote the expression of ZEB2 in thyroid cancer cells. Biochem Biophys Res Commun. 2018; 507: 168-72.

80. Xue C, Cheng Y, Wu J, et al. Circular RNA CircPRMT5 Accelerates Proliferation and Invasion of Papillary Thyroid Cancer Through Regulation of miR-30c/E2F3 Axis. Cancer Manag Res. 2020; 12: 3285-91.

81. Sun D, Chen L, Lv H, et al. Circ 0058124 Upregulates MAPK1 Expression to Promote Proliferation, Metastasis and Metabolic Abilities in Thyroid Cancer Through Sponging miR-940. Onco Targets Ther. 2020; Volume 13: 1569-81.

82. Xia F, Chen Y, Jiang B, et al. Hsa circ 0011385 accelerates the progression of thyroid cancer by targeting miR-361-3p. Cancer Cell Int. 2020; 20.

83. Yang Y, Ding L, Li Y, et al. Hsa_circ_0039411 promotes tumorigenesis and progression of papillary thyroid cancer by miR-1179/ABCA9 and miR-1205/MTA1 signaling pathways. J Cell Physiol. 2020; 235: 1321-29.

84. Pan Y, Xu T, Liu Y, et al. Upregulated circular RNA circ_0025033 promotes papillary thyroid cancer cell proliferation and invasion via sponging miR-1231 and miR-1304. Biochem Biophys Res Commun. 2019; 510: 334-38.

85. Zhang H, Ma XP, Li X, et al. Circular RNA circ_0067934 exhaustion expedites cell apoptosis and represses cell proliferation, migration and invasion in thyroid cancer via sponging miR-1304 and regulating CXCR1 expression. Eur Rev Med Pharmacol Sci. 2019; 23: 10851-66.

86. Wang M, Chen B, Ru Z, et al. CircRNA circ-ITCH suppresses papillary thyroid cancer progression through miR-22-3p/CBL/ $\beta$-catenin pathway. Biochem Biophys Res Commun. 2018; 504: 283-88.

87. Zhong $Y, \mathrm{Du} Y$, Yang $\mathrm{X}$, et al. Circular RNAs function as ceRNAs to regulate and control human cancer progression. Mol Cancer. 2018; 17: 79.

88. Liu Q, Pan LZ, Hu M, et al. Molecular Network-Based Identification of Circular RNA-Associated ceRNA Network in Papillary Thyroid Cancer. Pathol Oncol Res. 2020; 26: 1293-99.

89. Pemayun TG. Current Diagnosis and Management of Thyroid Nodules. Acta Med Indones. 2016; 48: 247-57.

90. Durante C, Grani G, Lamartina L, et al. The Diagnosis and Management of Thyroid Nodules: A Review. J Am Med Assoc. 2018; 319: 914-24.

91. Grani G, Lamartina L, Durante C, et al. Follicular thyroid cancer and Hürthle cell carcinoma: challenges in diagnosis, treatment, and clinical management. Lancet Diabetes Endocrinol. 2018; 6: 500-14.

92. Schiffman JD, Fisher PG, Gibbs P. Early detection of cancer: past, present, and future. Am Soc Clin Oncol Educ Book. 2015: 57-65.

93. Abdullah MI, Junit SM, Ng KL, et al. Papillary Thyroid Cancer: Genetic Alterations and Molecular Biomarker Investigations. Int J Med Sci. 2019; 16: $450-60$.

94. Shi E, Ye J, Zhang R, et al. A Combination of circRNAs as a Diagnostic Tool for Discrimination of Papillary Thyroid Cancer. Onco Targets Ther. 2020; 13: 4365-72.

95. Fan CM, Wang JP, Tang YY, et al. circMAN1A2 could serve as a novel serum biomarker for malignant tumors. Cancer Sci. 2019; 110: 2180-88.

96. Liu J, Zheng X, Liu H. Hsa_circ_0102272 serves as a prognostic biomarker and regulates proliferation, migration and apoptosis in thyroid cancer. J Gene Med. 2020: e3209.

97. Sun JW, Qiu S, Yang JY, et al. Hsa_circ_0124055 and hsa_circ_0101622 regulate proliferation and apoptosis in thyroid cancer and serve as prognostic and diagnostic indicators. Eur Rev Med Pharmacol Sci. 2020; 24: 4348-60.

98. Lan X, Cao J, Xu J, et al. Decreased expression of hsa_circ_0137287 predicts aggressive clinicopathologic characteristics in papillary thyroid carcinoma. J Clin Lab Anal. 2018; 32: e22573.

99. Wu G, Zhou W, Pan X, et al. Circular RNA Profiling Reveals Exosomal circ_0006156 as a Novel Biomarker in Papillary Thyroid Cancer. Mol Ther Nucleic Acids. 2020; 19: 1134-44.

100. Zhu L, Li XJ, Kalimuthu S, et al. Natural Killer Cell (NK-92MI)-Based Therapy for Pulmonary Metastasis of Anaplastic Thyroid Cancer in a Nude Mouse Model. Front Immunol. 2017; 8: 816.

101. Chow LQM. Head and Neck Cancer. N Engl J Med. 2020; 382: 60-72.

102. Licitra L, Mesía R, Keilholz U. Individualised quality of life as a measure to guide treatment choices in squamous cell carcinoma of the head and neck. Oral Oncol. 2016; 52: 18-23.

103. Yang D, Jin $Y$, Cheng $S$, et al. The interaction between circular RNA hsa_circ_0000285 and miR-599 in thyroid cancer. Eur Rev Med Pharmacol Sci. 2020; 24: 4882-89.

104. Liu F, Zhang J, Qin L, et al. Circular RNA EIF6 (Hsa circ 0060060) sponges miR-144-3p to promote the cisplatin-resistance of human thyroid carcinoma cells by autophagy regulation. Aging. 2018; 10: 3806-20.

105. Lee SI, Kim DK, Seo EJ, et al. Role of Krüppel-Like Factor 4 in the Maintenance of Chemoresistance of Anaplastic Thyroid Cancer. Thyroid. 2017; 27: 1424-32.

106. Jiménez-Fonseca P, Gómez Saez JM, Santamaria Sandi J, et al. Spanish consensus for the management of patients with anaplastic cell thyroid carcinoma. Clin Transl Oncol. 2017; 19: 12-20.

107. Hu S, Rayman MP. Multiple Nutritional Factors and the Risk of Hashimoto's Thyroiditis. Thyroid. 2017; 27: 597-610.

108. Guo $Y$, Zynat J, Xing S, et al. Immunological changes of $T$ helper cells in flow cytometer-sorted CD4(+) T cells from patients with Hashimoto's thyroiditis. Exp Ther Med. 2018; 15: 3596-602.

109. Kowalczyk A, Zegan M, Michota-Katulska E. Key minerals significant for hypothyroidism including Hashimoto's thyroiditis - function and presence in food. Wiad Lek. (Warsaw, Poland : 1960). 2017; 70: 778-83. 
110. Barić A, Brčić L, Gračan S, et al. Association of established hypothyroidismassociated genetic variants with Hashimoto's thyroiditis. J Endocrinol Invest. 2017; 40: 1061-67.

111. Xiong S, Peng H, Ding X, et al. Circular RNA Expression Profiling and the Potential Role of hsa_circ_0089172 in Hashimoto's Thyroiditis via Sponging miR125a-3p. Mol Ther Nucleic Acids. 2019; 17: 38-48.

112. Sun Y, Wang W, Tang Y, et al. Microarray profiling and functional analysis of differentially expressed plasma exosomal circular RNAs in Graves' disease. Biol Res. 2020; 53: 32.

113. Wu L, Zhou R, Diao J, et al. Differentially expressed circular RNAs in orbital adipose/connective tissue from patients with thyroid-associated ophthalmopathy. Exp Eye Res. 2020; 196: 108036.

114. Liu W, Zhao J, Jin M, et al. circRAPGEF5 Contributes to Papillary Thyroid Proliferation and Metastatis by Regulation miR-198/FGFR1. Mol Ther Nucleic Acids. 2019; 14: 609-16. 